\title{
A Fast and High-Accuracy Compass Alignment Method to SINS with Azimuth Axis Rotation
}

\author{
Xixiang Liu, Xiaosu Xu, Yiting Liu, and Lihui Wang \\ Key Laboratory of Micro-Inertial Instrument and Advanced Navigation Technology, School of Instrument Science \& Engineering, \\ Ministry of Education, Southeast University, Nanjing 210096, China \\ Correspondence should be addressed to Xixiang Liu; scliuseu@163.com
}

Received 30 September 2012; Accepted 10 January 2013

Academic Editor: Gerhard-Wilhelm Weber

Copyright (C) 2013 Xixiang Liu et al. This is an open access article distributed under the Creative Commons Attribution License, which permits unrestricted use, distribution, and reproduction in any medium, provided the original work is properly cited.

\begin{abstract}
Azimuth axis rotating modulation was introduced to improve the alignment accuracy of strapdown inertial navigation system (SINS) through compass algorithm, in which the limit accuracy was determined by equivalent sensor errors in the eastern and northern direction. In this modulation, horizontal sensor errors were modulated into zero mean periodic variables. Furthermore, two methods were introduced to ensure alignment accuracy and speed: (1) shortened rotating cycle and redesigned compass parameters were selected to eliminate or ease the amplification to low-frequency senor error inputs in compass loop caused by rotation and (2) a data repeated calculation method was designed to shorten prolonged alignment time caused by the above redesigned parameters. Based on a certain SINS, turntable test proves that alignment accuracy and time were significantly improved and slightly shortened in comparison with the classical compass alignment.
\end{abstract}

\section{Introduction}

Rotating modulation in the strapdown inertial navigation system (SINS), being successfully applied since 1980s, is an effective way to improve the accuracy of SINS with certain accuracy inertial sensors. In 1980, Levinson and Giovanni introduced a strapdown ship compass, in which uniaxial 4position stop program was used to compensate for the drifts of ring laser gyros, thus significantly improving the system accuracy [1]. Subsequently, Sperry developed MK-49 dualaxis rotating modulated laser SINS with 16-order rotating program and this system was selected as a standard ship's INS for the North Atlantic Treaty Organization (NATO) in 1989 $[2,3]$.

At the same time, theories about rotating modulation were concern. The widely accepted views about rotating modulation are as follows [1-4]: (1) with uniaxial rotation, symmetry sensor errors installed at the vertical direction to the rotating axis can be eliminated and (2) with biaxial rotation, all symmetry sensor errors can be eliminated. In 2007 and 2012, Yuan et al. proposed an improved 4-position program for uniaxial rotation and 16-order rotating program for biaxial rotation $[5,6]$, to further improve the system accuracy compared with the methods in the literatures of [13]. In existed researching files, some scholars aim to improve the navigation accuracy by rotating scheme designing [46] and others focus upon the added observability caused by rotation when the optimal estimation method based on Kalman filter is used for initial alignment [7-11]; however, less attention was put to the possibility of improving alignment accuracy of compass alignment method brought by rotating modulation. Therefore, apart from study on azimuth rotating modulation, more research goes to oscillation in horizontal alignment, divergence in azimuth, and time-consuming of alignment process and corresponding solutions were offered.

In SINS, through classical compass alignment method, the horizontal alignment accuracy is subjected to the equivalent horizontal accelerometer errors while the azimuth is subject to the equivalent eastern gyro error [11-14]. Applied in engineering, these equivalent errors are determined by senor errors and the carrier's attitude together. While with azimuth rotation, the horizontal sensor errors are modulated into zero mean periodic variables [5], which brings the possibility to improve the alignment accuracy of SINS through compass method. But the studies in this paper indicated an amplification effect in compass loop upon the inputs 


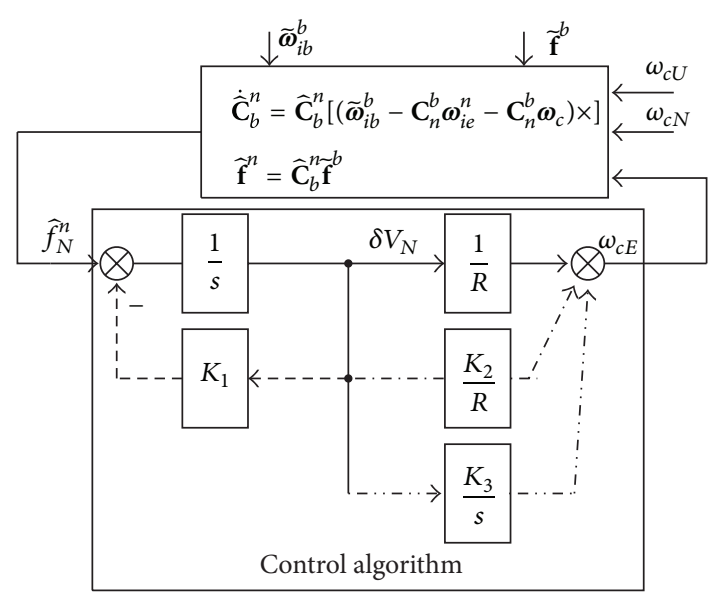

(a) Northern channel

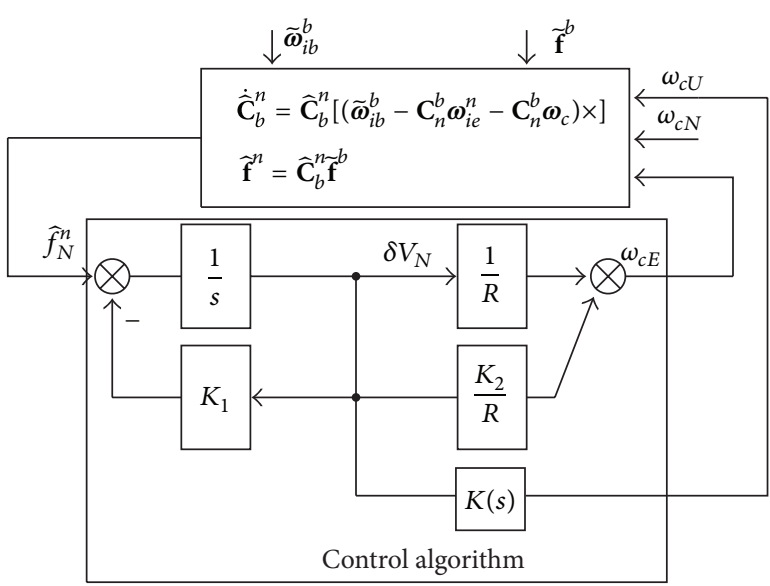

(b) Azimuth channel

FIGURE 1: Compass alignment loop in SINS.

of low-frequency sensor errors that are constant values if no rotation is involved. Such effect brought certain oscillation in horizontal alignment and divergence in azimuth. With the accelerated rotating speed and redesigned compass parameters, the amplification effect was eliminated or eased. Meanwhile, due to the above redesigned parameters, the alignment time was greatly prolonged.

To meet the requirement of SINS's rapid response, a fast alignment method based on data repeated calculation was designed. In this method, a characteristic of mathematical platform, cutting off the direct relationship between sensor data and feedback signals for alignment loop, in SINS is used, which is different from the relationship in PINS. With this characteristic and coupled with compass alignment control loop, the same set of data can be solved repeatedly to produce different direct cosine matrix (DCM) and different projected sensor data in mathematical platform from measured sensor data in body frame, thus producing different feedback signals. Moreover, a turntable test with one certain type of SINS was used to certify the effectiveness of these above methods.

The article is organized as follows: In Section 2, classical compass alignment to SINS will be introduced and simulation results will be given. In Section 3, azimuth rotating modulation will be introduced, simulation about compass alignment with rotation will be done, and the failure reasons will be analyzed; in the meantime, improved methods proved by simulation will be illustrated. In Section 4 , a fast compass alignment based on data repeated calculation will be put forward, and simulation about this method will be done. In Section 5, a turntable test with a certain type of SINS will be taken. Lastly, conclusions will be drawn in Section 6 .

\section{Classical Compass Alignment Algorithm to SINS}

2.1. Classical Compass Alignment Algorithm. With the development of SINS, compass alignment method has been widely used for its self-alignment. The main difference between
SINS and platform INS (PINS) lies in the application of mathematical platform in SINS while that of physical platform in PINS, that is to say, the sensor data in PINS are used as feedback signals while the projected sensor data in mathematical platform are used in SINS. But in terms of alignment process, the mechanisms are the same in both INSs.

Figures 1(a) and 1(b) are the northern and azimuth channels of compass fine alignment loop in SINS, respectively. And in Figure 1, $i, b$, and $e$ represent the inertial frame, the body frame, and the Earth frame, and the subscript $c$ represents the control signal from alignment loop, and the “ " and " $\sim$ " represent the calculation and measurement value of a vector respectively.

In Figure 1(a), the calculated value of DCM $\widehat{\mathrm{C}}_{b}^{n}$ contains the eastern misalignment $\phi_{x}$. With this DCM $\widehat{\mathbf{C}}_{b}^{n}$, Earth gravity $g$ will be projected into the calculated value of northern acceleration $\hat{f}_{N}^{n}$. When $\hat{f}_{N}^{n}$ is integrated, north velocity error $\delta V_{N}$ that can be set as a feedback signal in the northern channel to eliminate $\phi_{x}$ will be led. In this channel, the damping term $K_{1} s$ (dot lines) is applied to realize weak oscillation from equal amplitude oscillation, the forwardfeed term $K_{2} / R$ (dash-dot lines) is applied to shorten the oscillation period to $1 / \sqrt{1+K_{2}}$, and the integration term $K_{3} / s$ (double dash-dot lines) is introduced to eliminate the effect of $\phi_{z} \omega_{i e} \cos L+\varepsilon_{E}$.

In Figure 1(a), when the rapid and stable requirements of alignment process are comprehensively considered, control parameters can be designed by the following steps: (1) selecting the damping coefficient $\xi$ and the system adjusting time $T_{d}$, (2) calculating the damped oscillation angular rate $\omega_{n}=3 /\left(T_{d} \xi\right)$ and the attenuation coefficient $\sigma=\xi \cdot \omega_{n}$, and (3) calculating the control parameters as

$$
K_{1}=3 \sigma, \quad K_{2}=\frac{\sigma^{2}}{\omega_{s}^{2}}\left(2+\frac{1}{\xi^{2}}\right)-1, \quad K_{3}=\frac{\sigma^{3}}{g \xi^{3}},
$$

where $\omega_{s}$ is angular rate of Schuler. 
TABLE 1: Equivalent sensor errors in navigation frame.

\begin{tabular}{lccccc}
\hline Yaw $\left(^{\circ}\right)$ & 0 & 45 & 90 & 135 & 180 \\
\hline Accelerometer (ug) & & & & & \\
$\quad$ Eastern & 500 & 0 & -500 & -707.1068 & -500 \\
$\quad$ Northern & 500 & 707.1068 & 500 & 0 & -500 \\
$\quad$ Upper & 500 & 500 & 500 & 500 & 500 \\
Gyroscope $\left({ }^{\circ} / \mathrm{h}\right)$ & & & & & \\
$\quad$ Eastern & 0.05 & 0 & -0.05 & -0.0707 & -0.05 \\
$\quad$ Northern & 0.05 & 0.0707 & 0.05 & 0 & -0.05 \\
$\quad$ Upper & 0.05 & 0.05 & 0.05 & 0.05 & 0.05 \\
\hline
\end{tabular}

Similarly, in Figure 1(b), the parameters for azimuth channel can be calculated as

$$
K_{1}=K_{4}=2 \sigma, \quad K_{2}=\frac{4 \sigma^{2}}{\omega_{s}^{2}}-1, \quad K_{3}=\frac{4 \sigma^{4}}{g} .
$$

With the above parameters in (1) and (2), the limit accuracy of $\phi_{x}, \phi_{y}$ and $\phi_{z}$ can be achieved as follows:

$$
\begin{gathered}
\phi_{x s s}(s)=\lim _{s \rightarrow 0} s \phi_{x}(s)=-\frac{\nabla_{N}}{g}, \\
\phi_{z s s}(s)=\lim _{s \rightarrow 0} s \phi_{z}(s)=\frac{\varepsilon_{E}}{\omega_{i e} \cos L}, \\
\phi_{z s s}(s)=\lim _{s \rightarrow 0} s \phi_{z}(s)=\frac{\varepsilon_{E}}{\omega_{i e} \cos L},
\end{gathered}
$$

where $\omega_{i e}$ is the Earth angular rate, $L$ is the latitude, and $\nabla_{N}, \nabla_{E}$, and $\varepsilon_{E}$ are equivalent sensor errors in northern and eastern direction in the navigation frame.

In SINS equivalent errors in (3) are jointly determined by sensor errors and carrier's attitude, while those in PINS are determined by sensor errors. Such difference indicates that different carrier's attitude leads to different alignment accuracy by same sensor in SINS.

\subsection{Simulation}

(1) Simulation Setting. To set the system damping parameter $\xi$ as 0.707 and the adjusting time of horizontal loop $T_{d}$ as $150 \mathrm{~s}$, $K_{1}=0.06, K_{2}=1040.33306122, K_{3}=0.000081632653 \mathrm{can}$ be obtained. To set the adjusting time of azimuth loop $T_{d}$ as $300 \mathrm{~s}, K_{1}=K_{4}=0.02, K_{2}=259.3332653, K_{3}=4.082 e-$ -9 can be gotten. In alignment, the horizontal alignment process lasts for the first $150 \mathrm{~s}$ and the azimuth alignment covers the rest.

The horizontal attitudes of the carrier are set as $0^{\circ}$, the azimuth as $0^{\circ}, 45^{\circ}, 90^{\circ}, 135^{\circ}, 180^{\circ}$, respectively, all initial misalignment as $0^{\circ}$, and initial latitude and longitude as $32^{\circ}$ and $118^{\circ}$. The gyroscope constant and random errors are set as $0.05^{\circ} / \mathrm{h}$ and 0 , respectively, and the accelerometer constant and random errors as $500 \mathrm{ug}$ and 0 , respectively, and the height in navigation as 0 constantly. With above assumption,

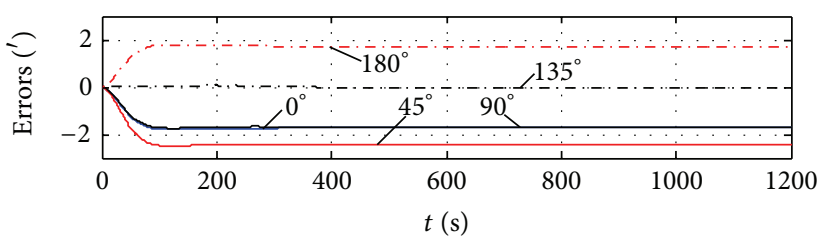

(a) Pitch

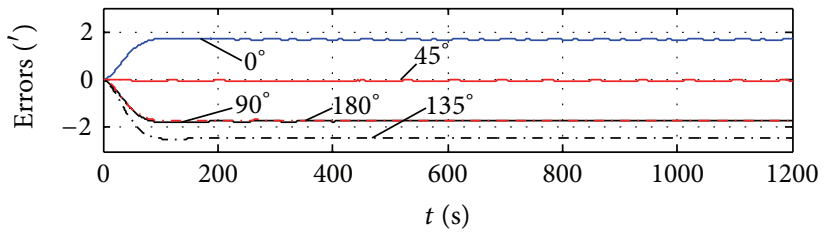

(b) Roll

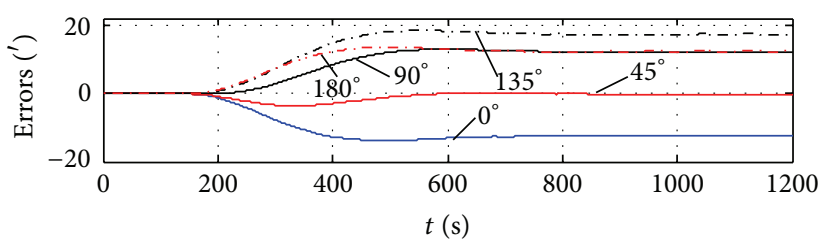

(c) Yaw

FIGURE 2: Alignment errors curves with classical compass alignment method.

the equivalent sensor errors in navigation frame $n$ can be calculated as shown in Table 1.

(2) Simulation Results. The simulation lasts $1200 \mathrm{~s}$, and simulation results are shown in Figure 2. The curves in Figure 2 indicate that about $200 \mathrm{~s}$ is needed to fulfill horizontal alignment and about $600-800 \mathrm{~s}$ for yaw. Statistics from $1000 \mathrm{~s}$ to $1200 \mathrm{~s}$ are shown in Table 2.

The curves in Figure 2 and statistics in Table 2 indicate that the alignment accuracy was jointly determined by sensor errors and carrier's attitude. In order to improve alignment accuracy with certain accuracy sensors and to eliminate the effect from the carrier's attitude, more methods are required.

\section{Compass Alignment Algorithm with Azimuth Axis Rotation}

3.1. Mechanism of Azimuth Rotational Modulation. In azimuth axis rotational modulation, the inertial measurement unit (IMU) is installed on the rotational platform that can rotate around the $z$-axis. Under this circumstance, the sensors measure the carrier's movement in the rotational frame $s$. Theses measured angular rate $\widetilde{\boldsymbol{\omega}}_{i s}^{s}$ and acceleration $\widetilde{\mathbf{f}}^{s}$ are supposed to be initially projected into the carrier body frame $b$ to participate in navigation solution:

$$
\begin{aligned}
\widehat{\boldsymbol{\omega}}_{i b}^{b} & =\mathbf{C}_{s}^{b}\left(\widetilde{\boldsymbol{\omega}}_{i s}^{s}+\boldsymbol{\omega}_{s b}^{s}\right), \\
\widehat{\mathbf{f}}^{b} & =\mathbf{C}_{s}^{b} \widetilde{\mathbf{f}}^{s} .
\end{aligned}
$$


TABLE 2: Statistics of alignment errors with classical compass alignment method.

\begin{tabular}{lccccc}
\hline Azimuth $\left(^{\circ}\right.$ ) & $0^{\circ}$ & $45^{\circ}$ & $90^{\circ}$ & $135^{\circ}$ & $180^{\circ}$ \\
\hline Pitch (minute sec) & & & & & \\
Limit accuracy & -1.7188 & -2.4308 & -1.7189 & 0 & 1.7189 \\
Actual value & -1.7215 & -2.4259 & -1.7215 & -0.0124 & 1.7092 \\
Roll (minute sec) & & & & & \\
Limit accuracy & 1.7188 & 0 & -1.7189 & -2.4308 & -1.7189 \\
Actual value & 1.7092 & 0.000003 & -1.7092 & -2.4259 & -1.7216 \\
Yaw (minute sec) & & & & & \\
Limit accuracy & -13.5124 & 0 & 13.5124 & 19.1065 & 13.5124 \\
Actual value & -12.3632 & -0.0157 & 12.3333 & 17.5139 & 12.4277 \\
\hline
\end{tabular}

Neglecting all other interferences and concerning sensor errors only, the above equation can be expanded as

$$
\begin{aligned}
\widehat{\boldsymbol{\omega}}_{i b}^{b} & =\mathbf{C}_{s}^{b}\left(\widetilde{\boldsymbol{\omega}}_{i s}^{s}+\boldsymbol{\omega}_{s b}^{s}\right)=\mathbf{C}_{s}^{b}\left(\boldsymbol{\omega}_{i s}^{s}+\boldsymbol{\varepsilon}^{s}+\boldsymbol{\omega}_{s b}^{s}\right) \\
& =\mathbf{C}_{s}^{b}\left(\boldsymbol{\omega}_{i b}^{s}+\boldsymbol{\omega}_{b s}^{s}+\boldsymbol{\varepsilon}^{s}+\boldsymbol{\omega}_{s b}^{s}\right)=\boldsymbol{\omega}_{i b}^{b}+\mathbf{C}_{s}^{b} \boldsymbol{\varepsilon}^{s} \\
\widehat{\mathbf{f}}^{b} & =\mathbf{C}_{s}^{b} \widetilde{\mathbf{f}}^{s}=\mathbf{C}_{s}^{b}\left(\mathbf{f}^{s}+\nabla^{s}\right)=\mathbf{f}^{b}+\mathbf{C}_{s}^{b} \nabla^{s} .
\end{aligned}
$$

Equation (5) indicates that the sensor errors have been modulated with rotation. When the rotation angular rate of rotational platform is set as $\omega$, the attitude matrix $\mathbf{C}_{b}^{s}$ can be constructed as

$$
\mathbf{C}_{s}^{b}=\left[\begin{array}{ccc}
\cos (\omega t) & -\sin (\omega t) & 0 \\
\sin (\omega t) & \cos (\omega t) & 0 \\
0 & 0 & 1
\end{array}\right] .
$$

Then $\boldsymbol{\varepsilon}^{b}=\mathbf{C}_{s}^{b} \boldsymbol{\varepsilon}^{s}$ and $\boldsymbol{\nabla}^{b}=\mathbf{C}_{s}^{b} \nabla^{s}$ can be expanded as

$$
\begin{aligned}
\varepsilon_{x}^{b} & =\cos (\omega t) \varepsilon_{x}^{s}-\sin (\omega t) \varepsilon_{y}^{s}, \\
\varepsilon_{y}^{b} & =\sin (\omega t) \varepsilon_{x}^{s}+\cos (\omega t) \varepsilon_{y}^{s}, \\
\varepsilon_{z}^{b} & =\varepsilon_{z}^{s}, \\
\nabla_{x}^{b} & =\cos (\omega t) \nabla_{x}^{s}-\sin (\omega t) \nabla_{y}^{s}, \\
\nabla_{y}^{b} & =\sin (\omega t) \nabla_{x}^{s}+\cos (\omega t) \nabla_{y}^{s}, \\
\nabla_{z}^{b} & =\nabla_{z}^{s} .
\end{aligned}
$$

The above equations in frame $b$ show that the sensor errors along $x$-axis and $y$-axis are modulated into zero mean periodic variables with azimuth axis rotation.

\subsection{Simulation for Classical Compass Alignment with Azimuth} Rotation. In SINS, the above modulated error in frame $b$ must be projected into frame $n$ with DCM $\mathbf{C}_{b}^{n}$ to get involved in the navigation solution, which will bring some negative effect to the above modulation. For ships or cars, the negative

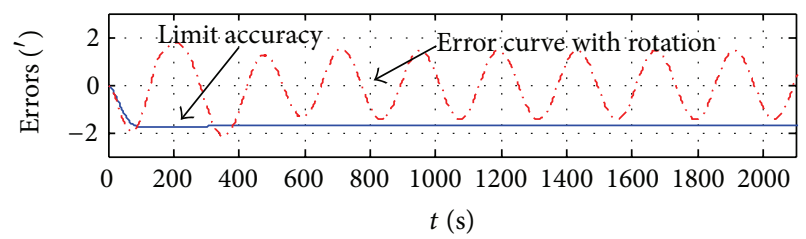

(a) Pitch

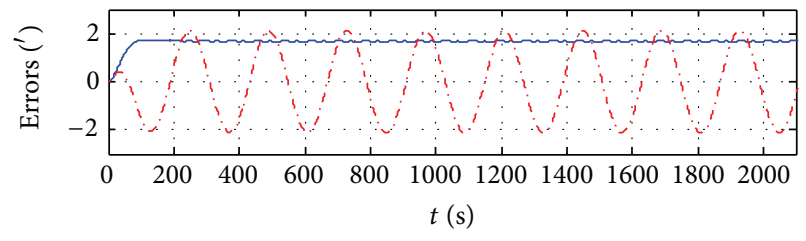

(b) Roll

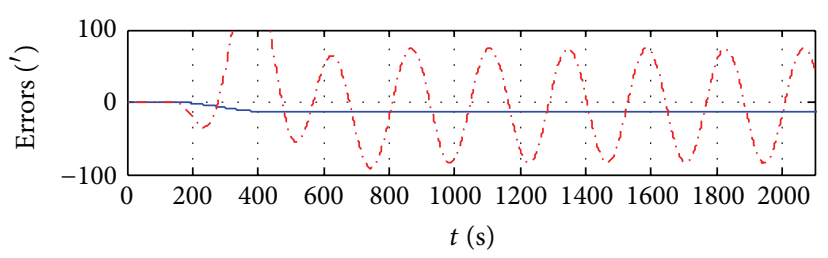

(c) Yaw

FIGURE 3: Alignment errors curves with the combination method.

effects caused by the above projection are limited because of their generally small horizontal attitude. Therefore, compared with the nonrotated modulation, the navigation accuracy of SINS can still be significantly improved.

Meanwhile, the above modulation brings the possibility to improve compass alignment accuracy. In the following simulation, azimuth rotation is brought into classical compass alignment method.

(1) Simulation Setting. The simulation is conducted under the following conditions: (1) the control loop parameters and the sensor errors set as same as those in Section 2.2, (2) the initial attitude and initial misalignments set as zero, (3) the rotating period of IMU set as $240 \mathrm{~s}$, and (4) no horizontal angle and linear movement.

(2) Simulation Results. The simulation lasts $2100 \mathrm{~s}$ and the simulation results are shown in Figure 3 as dot-dash lines. In Figure 3, the alignment results in Figure 2 with the carrier's attitude $0^{\circ}$ are redrawn in solid lines as a reference data. From Figure 3, conclusion can be drawn that the horizontal attitude error curves with azimuth rotation are oscillating with the period of $240 \mathrm{~s}$ and the oscillation amplitudes are approximate to the reference accuracy with nonrotation. The azimuth curve is oscillating with a period of $240 \mathrm{~s}$, but its oscillation amplitude is larger than that of nonrotation. Such a result indicates the failure of the simple combination of classical alignment and rotating modulation.

From Figure 3, the conclusion can be reached that negatively influential elements that result in the oscillation of horizontal alignment and failure of azimuth are sure to be found in the process of classical compass alignment with azimuth rotation. 


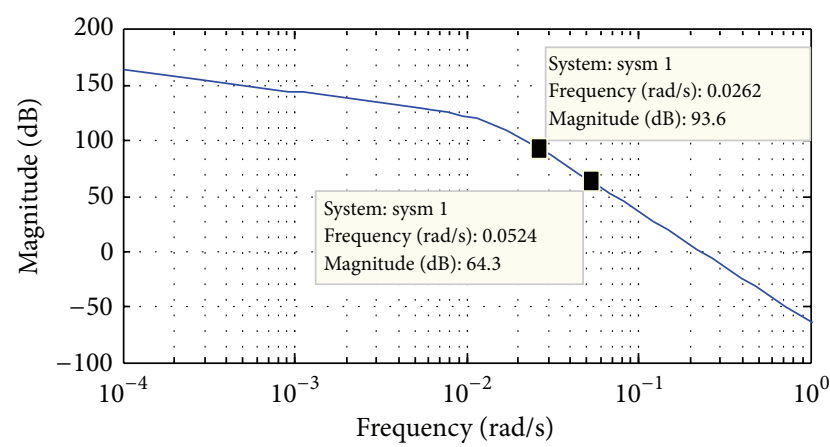

(a) TF1

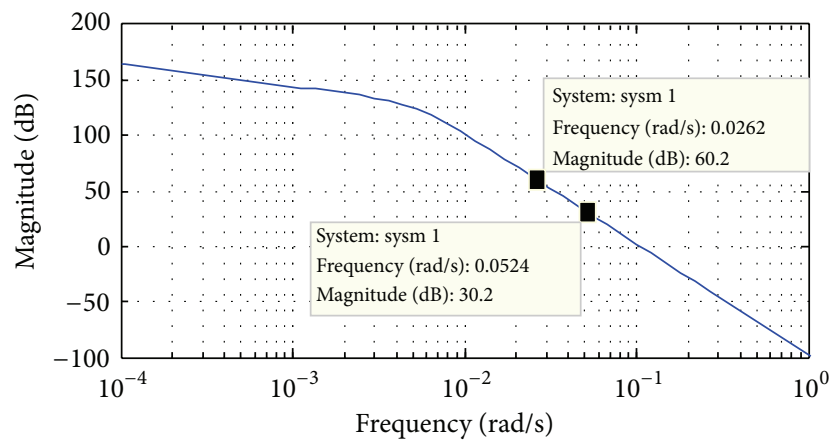

(c) TF1 with redesigned parameters

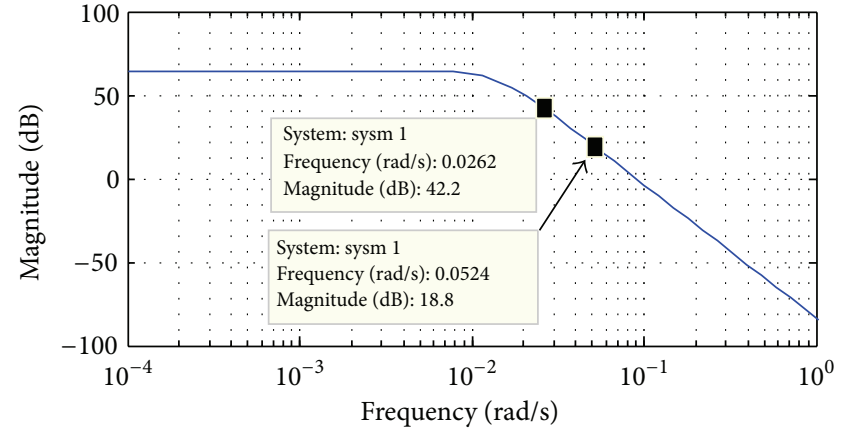

(b) TF2

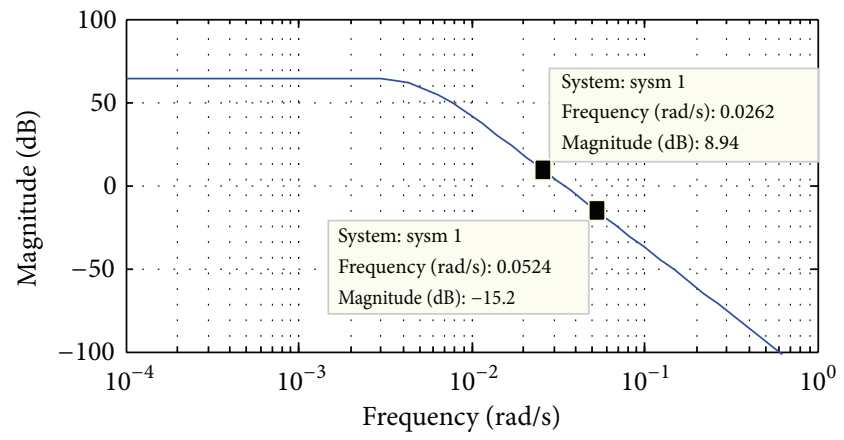

(d) TF2 with redesigned parameters

Figure 4: Bode charts.

\subsection{Further Analysis and Improved Methods}

3.3.1. The Reasons for Alignment Failure. Take the azimuth alignment loop as an example to analyze the reasons. In Figure 1(b), set $K(s)$ as $K_{3} /\left(\omega_{i e} \cos L\left(s+K_{4}\right)\right)$; then

$$
\begin{aligned}
\phi_{z}(s)= & \frac{s K(s)}{\Delta^{*}(s)}\left[\frac{\nabla_{N}(s)}{s}+\delta V_{N}(0)\right] \\
& +\frac{g K(s)}{\Delta^{*}(s)}\left[\frac{\varepsilon_{E}(s)}{s}+\phi_{x}(0)\right] \\
& +\frac{s\left(s+K_{1}\right)+\left(\left(K_{2}+1\right) / R\right) g}{\Delta^{*}(s)}\left[\frac{\varepsilon_{U}}{s}+\phi_{z}(0)\right],
\end{aligned}
$$

where $\Delta^{*}(s)=s^{3}+k_{1} s^{2}+\omega_{s}^{2}\left(K_{2}+1\right) s+\left(K_{3} /\left(s+K_{4}\right)\right) g$ and $\varepsilon_{U}$ is the equivalent upward gyro error.

With the azimuth axis rotation, the horizontal sensor errors are modulated into the periodically changing values; in other words, to the azimuth loop, the inputs are changed from constant values to periodically changing values.

According to (8), TF1 and TF2 are defined as transfer functions from the input $\left(\nabla_{N}(s) / s+\delta V_{N}(0)\right)$ and $\left(\varepsilon_{E}(s) / s+\right.$ $\left.\phi_{x}(0)\right)$ to the output $\phi_{z}(s)$. And the Bode charts of TF1 and TF2 with the parameters in Section 2.2 are shown in Figures 4(a) and 4(b), respectively.

In Figures 4(a) and 4(b), the magnification of the equivalent eastern gyro error is $93.6 \mathrm{~dB}$ and that of the equivalent northern accelerometer is $42.2 \mathrm{~dB}$ with the errors modulation period $240 \mathrm{~s}$ (the angular velocity is $0.0262 \mathrm{rad} / \mathrm{sec}$ ).
Therefore, alignment failure tends to result mostly from large magnification.

3.3.2. Improved Methods. To reduce the above magnification effect, two options can be selected: (1) to speed up the rotating angular rate, the input frequency will move from left to right in the Bode chart and (2) to adjust compass parameters will make the high-magnification range move from right to left part in Bode chart.

(1) Method One. $120 \mathrm{~s}$ is deemed as rotating cycle with respect to the increase of burden of mechanical indexing mechanism and other sensor errors followed by too fast rotating rate. Then the magnification of the equivalent eastern gyro error is $64.3 \mathrm{~dB}$ and that of the equivalent northern accelerometer is $18.8 \mathrm{~dB}$ as shown in Figures 4(a) and 4(b). The simulation results are presented in Figure 5 as with solid lines. Compared with the results in Figure 3, conclusion can be drawn that (1) the horizontal alignment errors are suppressed and (2) the azimuth error curve is oscillating and its oscillation amplitude is approximate to the reference value. Simulation results through this method indicate that the alignment accuracy is increased slightly.

(2) Method Two. In order to restrain the oscillation amplitude of horizontal alignment error and realize the convergence of azimuth alignment error, the adjusting time of horizontal loop is set as $600 \mathrm{~s}$ and that of azimuth is set as $800 \mathrm{~s}$. Then the parameters for horizontal loop are calculated as $K_{1}=0.015, K_{2}=64.0833$, and $K_{3}=5.10204 e-006$, and the those for azimuth are as $K_{1}=K_{4}=0.0075$, 


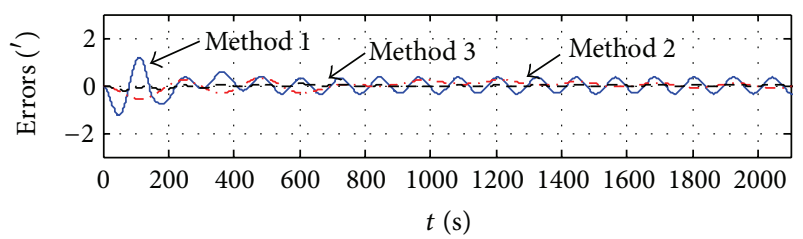

(a) Pitch

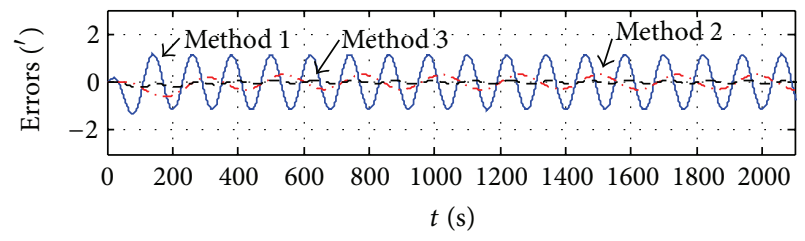

(b) Roll

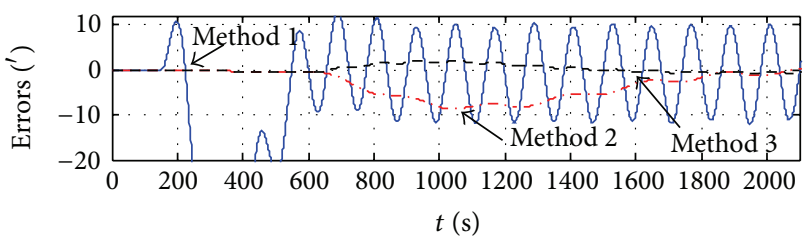

(c) Yaw

FIGURE 5: Alignment errors with different improved methods.

TABLE 3: Alignment errors with improved method 3.

\begin{tabular}{lcc}
\hline & Mean & Theoretical value \\
\hline Pitch (minute sec) & 0.0096 & -1.7188 \\
Roll (minute sec) & 0.0012 & 1.7188 \\
Yaw (minute sec) & -0.5603 & -13.5124 \\
\hline
\end{tabular}

$K_{2}=35.6094, K_{3}=8.07159 e-011$. Besides, the rotating period is still $240 \mathrm{~s}$. The horizontal alignment lasts for the first 600 s and the azimuth alignment lasts for the rest in alignment process. With these parameters, the magnification of the equivalent eastern gyro error is $60.2 \mathrm{~dB}$ and that of the equivalent northern accelerometer is $8.94 \mathrm{~dB}$ as shown in Figures 4(c) and 4(d). The simulation results are presented as dot-dash lines in Figure 5. Compared with those solid lines, the horizontal oscillation is obviously suppressed and its amplitude is decreased. The azimuth error curve converges slowly and tends to 0 , but the alignment time is extended to around $1900 \mathrm{~s}$.

(3) Method Three. In order to combine the advantages of the above two methods, $120 \mathrm{~s}$ is set as the rotating period and the alignment parameters are set as those in method 2. With the above parameters, the magnification of the equivalent eastern gyro error is $30.2 \mathrm{~dB}$ and that of the equivalent northern accelerometer is $-15.2 \mathrm{~dB}$ as shown in Figures 4(c) and $4(\mathrm{~d})$. The simulation results are presented as dotted line in Figure 5. Compared with those solid lines and dot-dash lines, the oscillation amplitude is further decreased and the convergence period of azimuth is shortened to about $1500 \mathrm{~s}$. It is confirmed that the combination of the above two methods is effective.

In Table 3, the mean data from $2000 \mathrm{~s}$ to $2100 \mathrm{~s}$ in method 3 are listed and the limit accuracy of compass algorithm without rotation when the carrier's attitudes are $0^{\circ}$ is also listed.

Compared results in Table 3 indicate that alignment accuracy can be significantly improved with azimuth rotation. But the curves in Figure 5(c) also show that the alignment time is too long.

In engineering, the alignment time may be longer due to the various interference factors and initial misalignments. This, for the sake of shortening alignment time, requires more available methods.

\section{A Fast Compass Alignment Method Based on Data Repeated Calculation}

4.1. A Possible Way to Accelerate Compass Alignment Speed of SINS. In Figure 6(a), inertial sensors are installed on the physical navigation platform in PINS and the difference between the sensor measured data and the ideal data from ideal navigation frame is decided by the misalignments. In other words, there are direct corresponding relationships between the sensor data and feedback correction signals.

While in SINS, inertial sensors are installed on the carrier and a mathematical platform is used to simulate the physical navigation platform as shown in Figure 6(b), in which $n^{\prime}$ is used to denote mathematical calculated frame from the calculated DCM $\widehat{\mathbf{C}}_{b}^{n}$. Before alignment, there are misalignments between $n^{\prime}$ and the ideal navigation frame $n$. Only after the sensor data obtained from frame $b$ is projected into frame $n^{\prime}$ can it get involved in compass alignment operation. That is, no direct corresponding relationship between the sensor data and feedback signals is found. On such condition different attitude matrixes $\widehat{\mathbf{C}}_{b}^{n}$ determine different projection data in frame $n^{\prime}$ and further determine the feedback signals with the same sensor data.

This difference between the compass methods in SINS and PINS indicates that a same set of data collected in frame $b$ can be used to fulfill alignment by the repeated calculation for $\widehat{\mathbf{C}}_{b}^{n}$, that is to say, sensor data collecting time will be reduced and alignment time will be shortened with use of computer's high-speed performance at most.

\subsection{Repeated Calculation Algorithm in SINS}

4.2.1. Navigation Resolution Algorithm in SINS. The recurrence equations for SINS navigation resolution can be expressed as

$$
\begin{gathered}
\mathbf{C}_{b k}^{n}=\mathbf{C}_{b k-1}^{n}\left(\mathbf{I}+T_{s} \boldsymbol{\omega}_{n b k}^{b} \times\right), \\
\mathbf{V}_{k}^{n}=\mathbf{V}_{k-1}^{n} \\
+T_{s}\left[\mathbf{C}_{b k-1}^{n} \mathbf{f}_{k}^{b}-\left(2 \boldsymbol{\omega}_{i e k-1}^{n}+\boldsymbol{\omega}_{e n k-1}^{n}\right) \times \mathbf{V}_{k-1}^{n}+\mathbf{g}^{n}\right], \\
L_{k}=L_{k-1}+\frac{T_{s} V_{N k-1}^{n}}{R_{M}+h_{k-1}}, \quad \lambda_{k}=\lambda_{k-1}+\frac{T_{s} V_{E k-1}^{n} \sec L_{k-1}}{R_{N}+h_{k-1}}, \\
h_{k}=h_{k-1}+T_{s} V_{U k-1}^{n},
\end{gathered}
$$




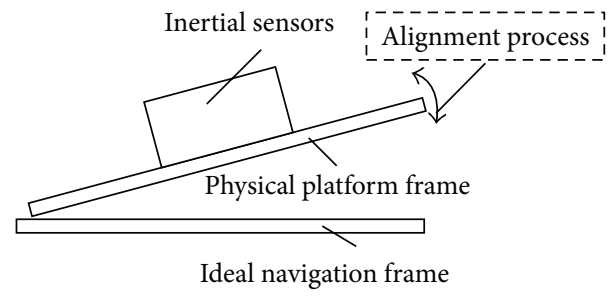

(a)

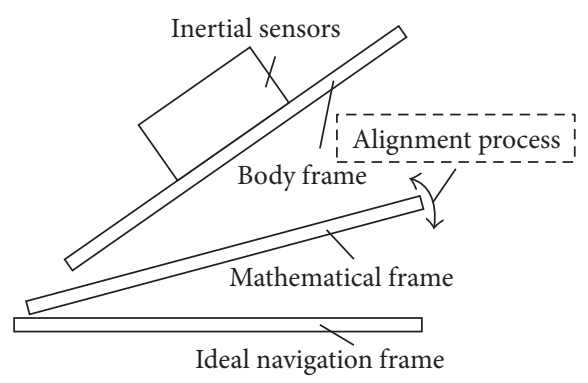

(b)

FIGURE 6: Alignment process for PINS and SINS.

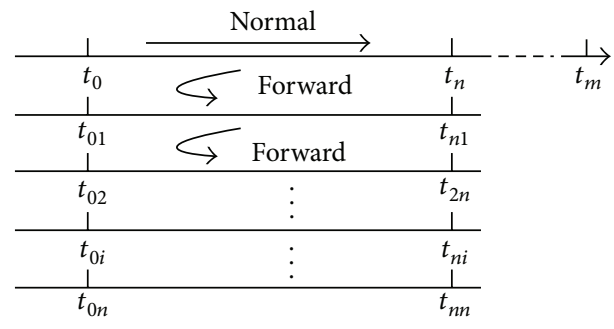

FIGURE 7: Repeated calculation algorithm.

where $k$ is recursive number, $T_{s}$ is the sensor data sampling and navigation resolution update cycle, $\boldsymbol{\omega}_{n b}^{b}=\boldsymbol{\omega}_{i b}^{b}-$ $\left(\mathbf{C}_{b}^{n}\right)^{T}\left(\boldsymbol{\omega}_{i e}^{n}+\boldsymbol{\omega}_{e n}^{n}\right), \boldsymbol{\omega}_{e n}^{n}=\left[-V_{N}^{n} / R V_{E}^{n} / R\left(V_{E}^{n} \tan L\right) / R\right]^{T}$, " $A_{B C}^{D}$ " denotes the projection of a motion vector $\mathbf{A}$ that means the relative motion from $C$ to frame $B$ frame in $D$ frame, $\mathbf{C}_{b}^{n}$ is the DCM, $\mathbf{V}^{n}=\left[\begin{array}{lll}V_{E}^{n} & V_{N}^{n} & V_{U}^{n}\end{array}\right]$ is the velocity vector, $L, \lambda$, and $h$ are the latitude and longitude and height, respectively and $(\cdot x)$ denotes the antisymmetric matrix of the vector ".". For ships and cars, the height can be set as zero constantly.

\subsubsection{A Repeated Calculation Algorithm for Compass Align-} ment. The above equations (9) constitute the basic update equations of navigation calculation. The sensor data are of the "real-time" meaning when involved in the recursion operation or losing their meaning in the next period after the completion of the current navigation calculation. But as the analysis in Section 4.1, initial alignment of SINS can be completed by the repeated calculation for $\widehat{\mathbf{C}}_{b}^{n}$ with a set of data.

When the carrier is in the angular but nonline motion, which is common state for compass alignment, a repeated calculation algorithm is designed as shown in Figure 7.

The normal collection for sensor data and normal navigation calculation is done in the period from $t_{0}$ to $t_{n}$ and the sensor data is stored at the same time. In addition, the forward repeated calculation is done in the subsequent period from $t_{n}$ to $t_{m}$ or the period from $t_{01}$ to $t_{n n}$ according to Figure 7 .

In the period from $t_{01}$ to $t_{n 1}, t_{01}$ is the starting time and the forward calculation has the same calculation process as in the normal navigation resolution. If the carrier is in nonline motion, the velocity and the position of it at $t_{n}$ can be set as the initial velocity and the position at $t_{01}$. However, the attitude at $t_{01}$ differs from that at $t_{n}$ by reason of its angular movement. What's more, an instantaneous solidification body frame is introduced to take the carrier body frame at $t_{0}$ as the solidification body frame $i_{b_{0}}$, which means $\mathbf{C}_{b}^{i_{b_{0}}}\left(t_{0}\right)=\mathbf{I}$. Then, the $\mathrm{C}_{b}^{i_{b_{0}}}(t)$ can be updated by gyro data:

$$
\mathbf{C}_{b k}^{i_{b_{0}}}=\mathbf{C}_{b k-1}^{i_{b_{0}}}\left(\mathbf{I}+T_{s} \boldsymbol{\omega}_{i_{b 0} b k}^{b} \times\right)=\mathbf{C}_{b k-1}^{i_{b_{0}}}\left(\mathbf{I}+T_{s} \boldsymbol{\omega}_{i b k}^{b} \times\right) .
$$

With (10), initial DCM at $t_{01}$ can be obtained by the matrixes $\mathbf{C}_{b}^{n}\left(t_{n}\right)$ and $\mathbf{C}_{b}^{i_{b_{0}}}\left(t_{n}\right)$ :

$$
\mathbf{C}_{b}^{n}\left(t_{10}\right)=\mathbf{C}_{b}^{n}\left(t_{n}\right)\left(\mathbf{C}_{b}^{i_{b_{0}}}\left(t_{n}\right)\right)^{T} .
$$

In the period from $t_{0 i}$ to $t_{n i}$, similar processes can be conducted as the period from $t_{01}$ to $t_{n 1}$. With the above process, in the period from $t_{0 i}$ to $t_{n i}$, the vehicle can move from the origin to the end, just as that in the period from $t_{0}$ to $t_{n}$.

4.2.3. Real-Time Attitude Calculation at the End of Repeated Calculation. When the above repeated calculation ends, calculated DCM is for the time $t_{n}$, not for the time $t_{m}$. In order to ensure the real-time property of SINS navigation, DCM for the time $t_{m}$ must be obtained. To meet this requirement, solidification body frame in Section 4.2.2 is also used. A new instantaneous solidification body frame $i_{b_{1}}$ is defined at time $t_{n}$ and $\mathbf{C}_{b}^{i_{b_{1}}}(t)$ can be updated with gyro data. Therefore, when repeated calculation ends, the real-time DCM can be obtained as

$$
\mathbf{C}_{b}^{n}\left(t_{m}\right)=\mathbf{C}_{b}^{n}\left(t_{n n}\right) \mathbf{C}_{b}^{i_{b_{1}}}\left(t_{m}\right) .
$$

\subsection{Simulation for Compass Alignment Based on Repeated Calculation}

(1) Simulation Setting. The simulation is carried out with the following conditions: (1) the gyro constant and random errors set as $0.05^{\circ} / \mathrm{h}$ and the accelerometer constant error and random errors as $500 \mathrm{ug}$, (2) the amplitude of pitch, rolling, and azimuth set as $2^{\circ}, 5^{\circ}$, and $2.5^{\circ}$ and the swing period of pitch rolling, and azimuth as $4 \mathrm{~s}, 6 \mathrm{~s}$, and $4 \mathrm{~s}$, respectively, (3) 


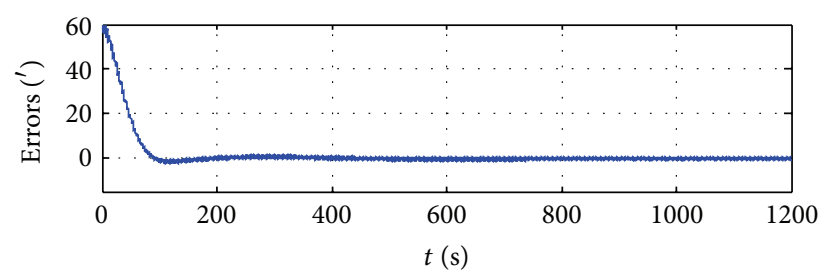

(a1) Pitch

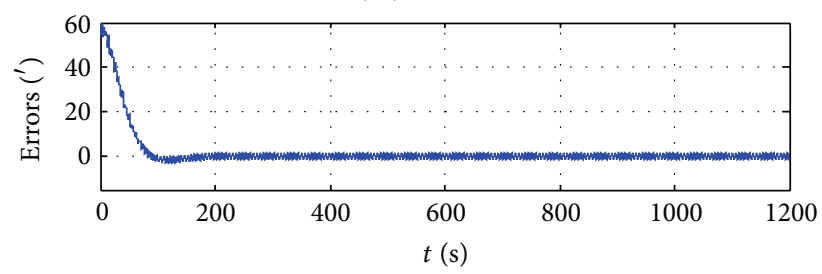

(b1) Roll

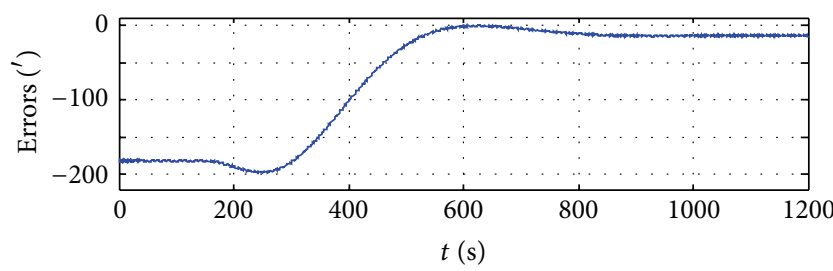

(c1) Yaw

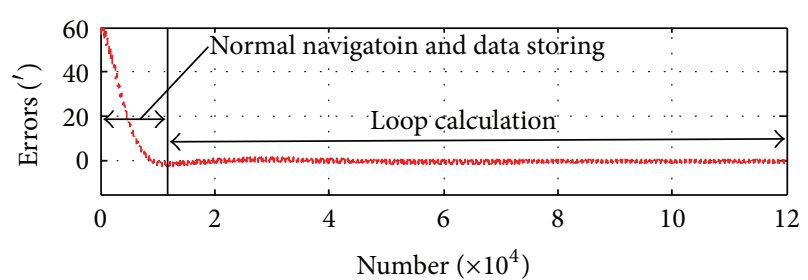

(a2) Pitch

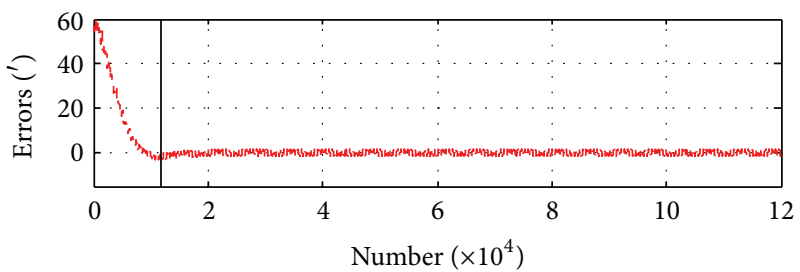

(b2) Roll

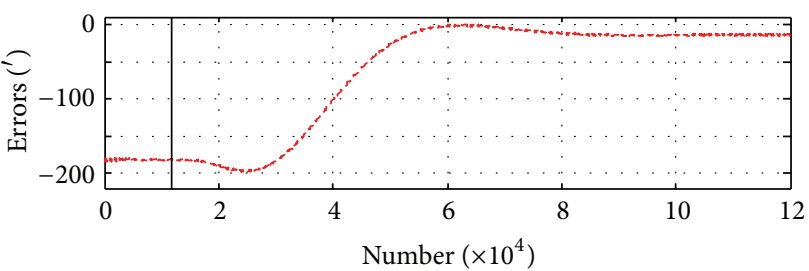

(c2) Yaw

FIGURE 8: Compass alignment with and without repeated calculation.

the initial azimuth of carrier set as 0 and its initial horizontal attitude as 0 , (4) the initial misalignment of pitch, rolling and azimuth set as $-1^{\circ},-1^{\circ}$, and $3^{\circ}$, respectively, (5) the initial velocity and acceleration set as 0 with its initial latitude and longitude as $32^{\circ}$ and $118^{\circ}$, respectively, (6) the initial height as 0 and the height set as 0 constantly in the calculation, (7) the first $120 \mathrm{~s}$ data stored to do the repeated calculation $(120 \mathrm{~s}$ cycle period), and (8) the compass loop parameters set as those in Section 2.2 and the update cycle as $10 \mathrm{~ms}$.

(2) Simulation Results. The simulation lasts 1200 s, during which two different simulation scheme results are compared. Moreover, the second scheme is based on classical compass alignment with data repeated calculation while the first one is not. In Figure 8, (al), (bl), and (cl) are the alignment error of pitch, roll, and yaw of Scheme 1, respectively, and those of Scheme 2 are likewise.

In Figure 8, the curves in (a1) and (a2), (b1) and (b2), and (c1) and (c2) present the same trends. In other words, the effect of the compass alignment based on the date repeated calculation matches that of the classical compass alignment. Thus, alignment time can be shortened by conducting the repeated calculation to a set of sensor data with high-speed computer calculation.

\section{Turntable Test}

Based on the azimuth axis rotating modulation and repeated calculation, the fast and high-accuracy compass alignment algorithm of SINS proposed in this paper has been verified
TABLE 4: Sensor precision of SINS.

\begin{tabular}{llll}
\hline \multicolumn{2}{c}{ Gyro } & & \multicolumn{2}{c}{ Accelerometer } \\
\hline Constant errors & $<0.05^{\circ} / \mathrm{h}$ & Constant errors & $\pm 5 \times 10^{-5} \mathrm{~g}$ \\
Random errors & $<0.2^{\circ} / \mathrm{h}$ & Random errors & $<5 \times 10^{-3} \mathrm{~g}$ \\
\hline
\end{tabular}

by the triaxial swing turntable test. The test was run as semiphysical simulation with data collected from the turntable and SINS.

5.1. Test Condition. In this test, the rate controlling accuracy of the turntable is $\pm 0.0005^{\circ} / \mathrm{s}$ and angle measuring accuracy is $\pm 0.0001^{\circ} / \mathrm{s}$.

Developed by Southeast University, flexible gyros and quartz accelerometers are used in this type of SINS. The accuracy of gyros and accelerometers are provided in Table 4 . Besides, the frequency of navigation computer is $333 \mathrm{MHz}$. The IMU is mounted on the turntable as shown in Figure 9. The update frequency of the turntable data and the SINS sensor data are $100 \mathrm{~Hz}$. The navigation solution frequency of SINS is $100 \mathrm{~Hz}$ as well.

Sensors' zero bias, scale factor, coupling coefficient, installing error, and the variable of the flexible gyro related to $g$ can be calculated by the exact calibration [15]. A conclusion can be gotten that no above-mentioned errors exist and the table frame is overlapped with the sensor frame if the test is conducted followed by the calibration. In order to simulate the sensor errors caused by a long time of conservation and 


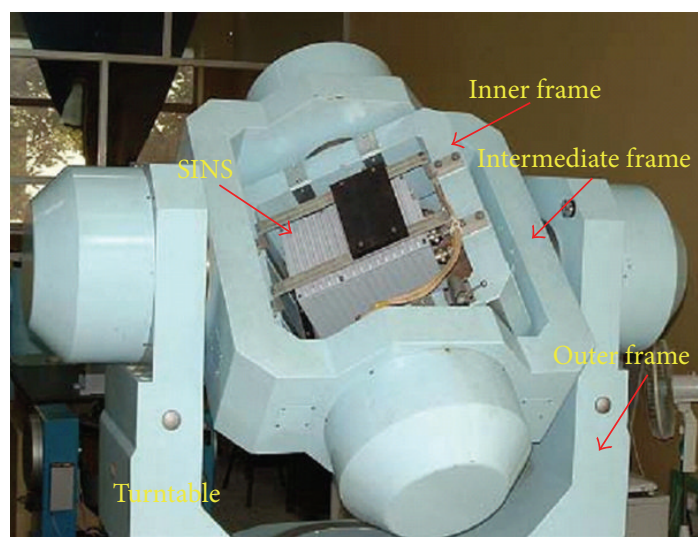

FIGURE 9: The turntable and SINS.

TABLE 5: Setting of sensors' error.

\begin{tabular}{lcc}
\hline & Gyro $\left({ }^{\circ} / \mathrm{h}\right)$ & Accelerometer $\left(\mathrm{m} / \mathrm{s}^{2}\right)$ \\
\hline$x$-axis & -0.070 & 0.01 \\
$y$-axis & 0.110 & -0.01 \\
$z$-axis & 0.100 & 0.01 \\
\hline
\end{tabular}

sensor unsuccessive turning-on error, the sensors' errors are artificially added as shown in Table 5 after the calibration.

5.2. Description of Test Process. The rotating period is set as 120 s and the parameters for compass loop as those of in Section 3.3.2.

During the test, the inner frame and the intermediate frame of the turntable are kept in level states constantly. At $t_{0}$, the outer frame rotates to $35^{\circ}$ (this value can be selected randomly); then take the table frame $s$ at this time as the carrier body frame $b_{0}$, which means the carrier theoretical attitude value is $\left[\begin{array}{lll}0 & 0 & 35^{\circ}\end{array}\right]$. At $14.6 \mathrm{~s}$, the turntable begins to rotate by $10^{\circ} / \mathrm{s}^{2}$ angular rate acceleration to get the angular rate $3 \%$. After $1.4 \mathrm{~s}$, the angular rate is stable enough. Moreover, at the moment of $16 \mathrm{~s}$ is defined as $t_{1}$ and the table as frame $b_{1}$.

During the test, the incremental angle information from turntable is adopted and the angular rate is considered as a constant value. Under such condition, analytical coarse alignment [7] is executed to get the DCM $\widetilde{\mathbf{C}}_{b_{0}}^{n}$ before the turntable starts to rotate afterwards; incremental angle of turntable from $t_{0}$ to $t_{1}$ is used to calculate DCM $\widetilde{\mathbf{C}}_{b_{1}}^{b_{0}}$, and thus the initial DCM at $t_{1}$ can be calculated as $\widetilde{\mathbf{C}}_{b_{1}}^{n}=\widetilde{\mathbf{C}}_{b_{0}}^{n} \widetilde{\mathbf{C}}_{b_{1}}^{b_{0}}$. When compass alignment based on repeated calculation ends, fine DCM $\widetilde{\mathbf{C}}_{b_{1}}^{n}$ can be gotten and fine DCM $\widetilde{\mathbf{C}}_{b_{0}}^{n}$ can be calculated as $\widetilde{\mathbf{C}}_{b_{0}}^{n}=\widetilde{\mathbf{C}}_{b_{1}}^{n}\left(\widetilde{\mathbf{C}}_{b_{1}}^{b_{0}}\right)^{T}$.

5.3. Test Results and Discussion. Two compass alignment schemes are compared with data repeated calculation applied in Scheme 1 instead of Schema 2. Data from $0 \mathrm{~s}$ to $14.4 \mathrm{~s}$ are used for analytical coarse alignment in both schemes. In Scheme 1, real-time navigation solution and compass alignment are run from $16 \mathrm{~s}$ to $136 \mathrm{~s}$, with the data in this period used for repeated calculation. After $136 \mathrm{~s}$, the 19 cycles of repeated calculations and compass alignment are completed. In Scheme 2, real-time navigation solution and compass alignment are run from $16 \mathrm{~s}$ to $2416 \mathrm{~s}$. However, the update times of both navigation solution and alignment operation are 240000. The alignment results come to the DCM $\widetilde{\mathbf{C}}_{b_{1}}^{n}$ and the true attitude $\left[\begin{array}{lll}0 & 0 & 37.5287^{\circ}\end{array}\right]$ can be obtained from turntable output.

5.3.1. Analysis of Sensor Data. The process of recording the turntable data and sensor data takes about 3700 s. Solid line and dotted line represent sensor data and projected data from frame $s$ to the frame $b_{1}$ both in the time of $16 \mathrm{~s}$ to $3516 \mathrm{~s}$, respectively, in Figure 10. Therefore, with azimuth rotation, sensor constant errors along $x$-axis and $y$-axis are modulated into zeros mean periodic variables while sensor error along $z$-axis is not modulated. Apart from this, the gyro output caused by turntable rotation is removed after projection from frame $s$ to $b_{1}$, but gyro constant error still exists as presented in Figure 10(c).

5.3.2. Alignment Accuracy. In accordance with alignment results in Figure 11, the enlarged portions are the attitude curves of the last repeated calculation cycle for Scheme 1 and of the last rotation cycle for Scheme 2, respectively.

In Figure 11, the true attitude and the limit attitude are represented by dotted lines and dot-dash lines. With the true value of $\mathrm{DCM} \mathrm{C}_{b_{1}}^{n}$ and sensor error in Table 5, equivalent northern and eastern accelerometer errors in $n$ frame are $0.0140 \mathrm{~m} / \mathrm{s}^{2}$ and $-0.0020 \mathrm{~m} / \mathrm{s}^{2}$ respectively and equivalent eastern gyro is $-0.1221^{\circ} / \mathrm{s}$; thus the limit accuracy can that be gotten by classical compass alignment without rotation is $\left[0.0819^{\circ}-0.0115^{\circ}-0.5500^{\circ}\right]$; in other words, the limit attitude is $\left[0.0819^{\circ}-0.0115^{\circ} 36.9787^{\circ}\right]$.

As analyzed in Section 2, the equivalent sensor errors are determined by sensor errors and the carrier's attitude in SINS. On the above condition, the equivalent error of northern accelerometer is relatively small and that of eastern gyro is relatively large. Due to this, the accuracy of classical compass alignment method cannot objectively reflect the effects of sensor errors to the alignment accuracy. Assume that the carrier's attitude is 0; then the limit accuracy obtained from classical compass alignment without rotation is $\left[0.0585^{\circ}-0.0585^{\circ}-0.3152^{\circ}\right]$, that is to say, limit attitude is $\left[0.0585^{\circ}-0.0585^{\circ} 37.2135^{\circ}\right]$. In Figure 11, this limit attitude is shown as solid lines.

The mean, variance, and peak-peak values from no. 229600 to no. 241600 of Scheme 1 and 2296 s to $2416 \mathrm{~s}$ of Scheme 2 are listed in Table 6 . The statistical results indicate that the alignment accuracy is significantly improved in both schemes.

In Table 6, compared with that acquired from classical compass alignment method, which is $\left[0.0585^{\circ}-0.0585^{\circ}-0.3152^{\circ}\right]$, the horizontal alignment accuracy is improved by 6 times and the azimuth alignment accuracy is improved by 8 times, if the mean attitude of Scheme 1 is chosen as alignment result, which is [0.00855 $0.00812-0.0336]$. 


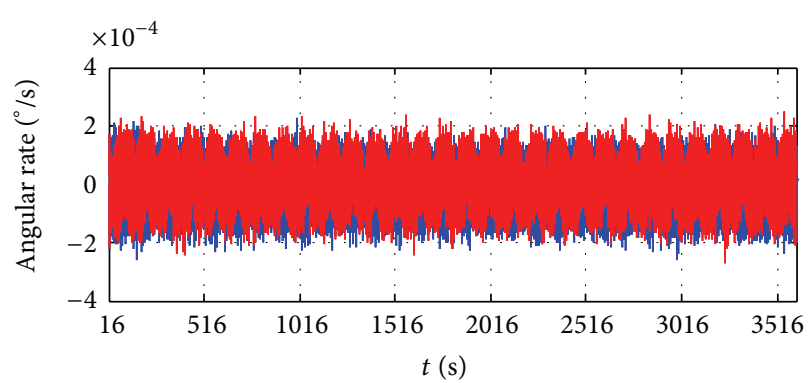

(a)

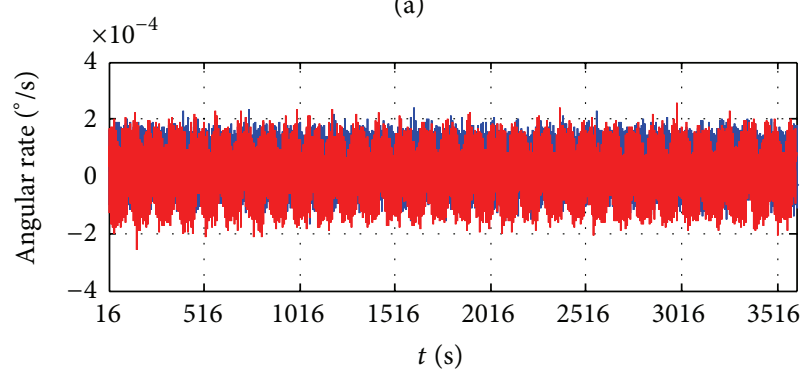

(b)

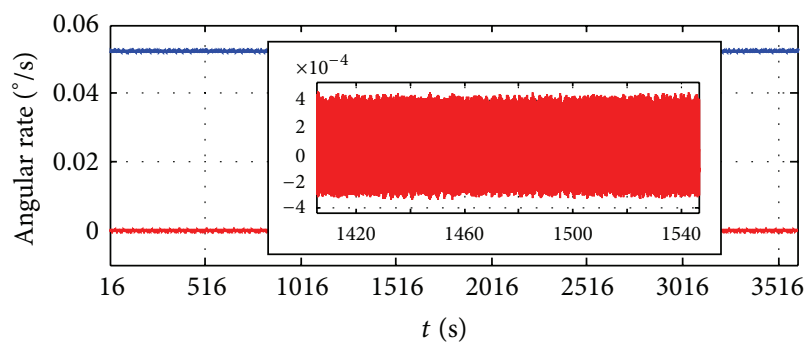

(c)

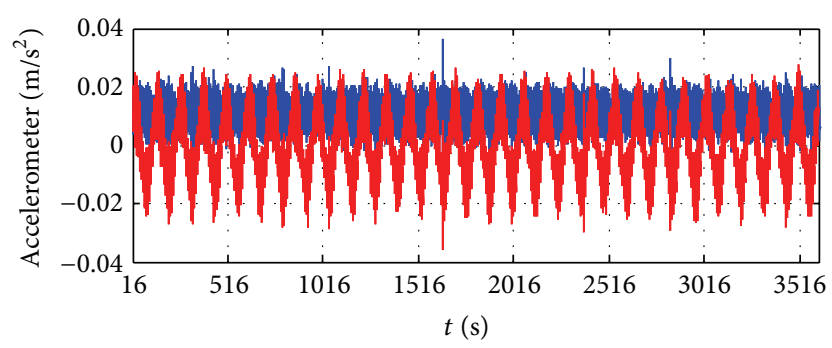

(d)

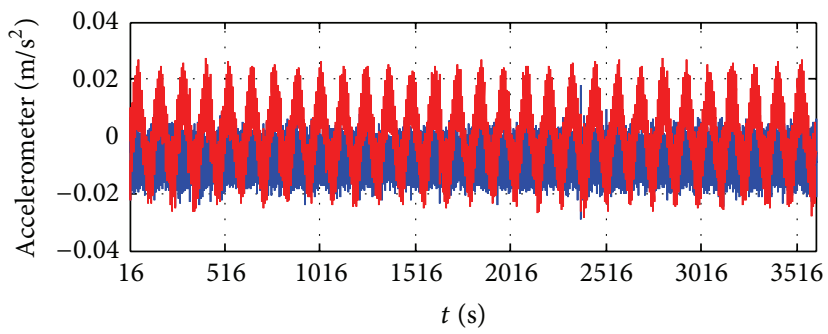

(e)

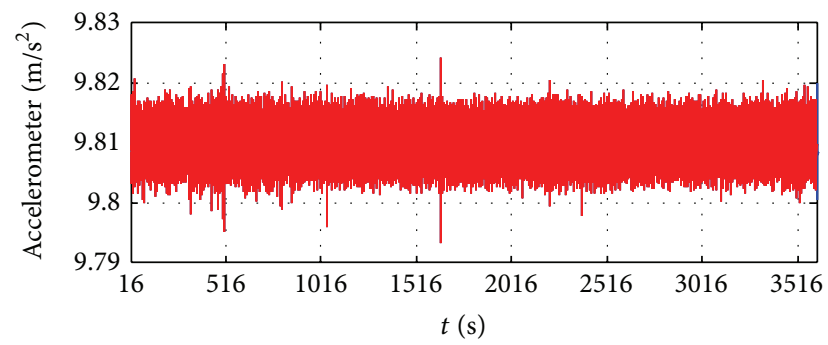

(f)

FIgURE 10: Senor data and their projections in frame $b_{1}$.

In Table 6, compared with the limit alignment error, the horizontal alignment accuracy is improved by 1 time and the azimuth alignment accuracy is improved by 4 times, if the maximum/minimum values of the alignment curves of Scheme 1 is chosen as alignment result, which is $\left[0.0291^{\circ} \%-0.0146^{\circ} 0.0288^{\circ} /-0.0144^{\circ} 0.0614 \%{ }^{\circ} / 0.0202^{\circ}\right]$.

With the fine alignment result of $\mathrm{DCM} \widehat{\mathbf{C}}_{b_{1}}^{n}$ and DCM $\widetilde{\mathbf{C}}_{b_{1}}^{b_{0}}$, DCM $\widehat{\mathbf{C}}_{b_{0}}^{n}$ can be calculated.

5.3.3. Alignment Time. In this compass alignment, which is based on azimuth rotation and data repeated calculation, the alignment time is determined by the performance of the navigation computer after coarse alignment, the accelerated rotation, and data collection in one modulation cycle. As for a computer with $333 \mathrm{MHz}$ frequency about $1 \mathrm{~ms}$ is required for a single navigation solution and compass alignment operation. To fulfill 22800 update operations (19 cycles of repeated calculations) $228 \mathrm{~s}$ is needed. The whole process for alignment takes $364 s(16 s+120 s+228 s)$ which is superior to the classical alignment time (600-800 s) as shown in Section 2.2.

In engineering, the conflict among the real-time sen-

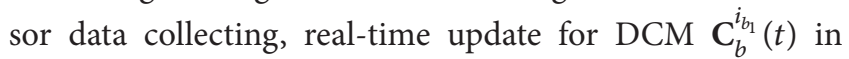

Section 4.2.3, and the repeated calculation can be resolved by multi-tasking real-time operating system (RTOS). In this type of SINS, VxWorks, an RTOS, is applied. When RTOS' spending time for tasking scheduling and the time consumed for $\mathbf{C}_{b}^{i_{b_{1}}}(t)$ are concerning, the total consumed time for alignment is a little longer than $364 \mathrm{~s}$ and the measured time in the above SINS is $402 \mathrm{~s}$.

\section{Conclusions}

In this paper, in order to improve the alignment accuracy with certain accuracy sensors and eliminate the effect of carrier's attitude to alignment accuracy, azimuth rotating modulation method is introduced to classical compass alignment for SINS.

Two methods are adopted to cope with the amplification effort in classical compass loop to low-frequency input, which does not exist without rotating modulation. The first method is to increase the rotating frequency and the second is to redesign the parameters of compass loop. The simulation results indicate that alignment accuracy is significantly increased through the above two methods; however, results turn out that alignment time is too long to be accepted. 


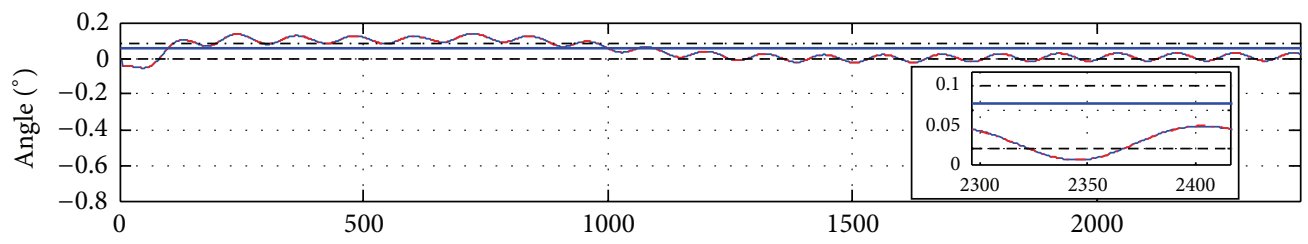

... - True value

- - - Limit attitude for attitude $b_{1}$

— Limit attitude for attitude 0

Inserted figure

- Scheme 1 number $(\times 100)$

-. - Scheme $2 t(\mathrm{~s})$

(a) Pitch

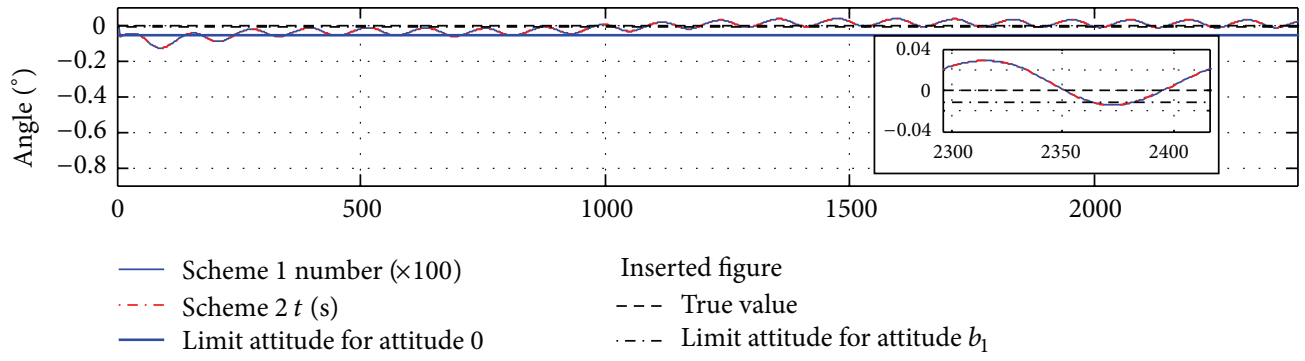

(b) Roll

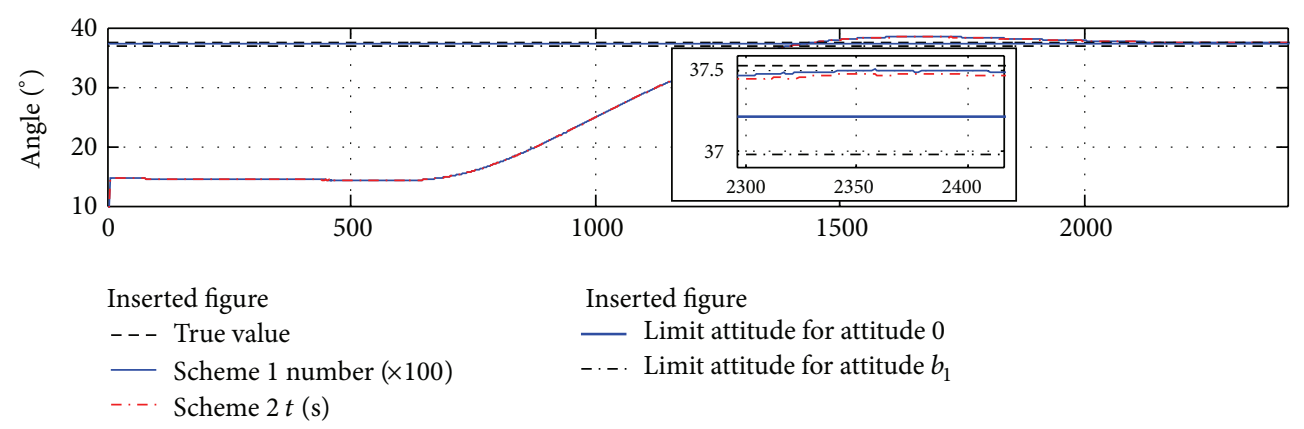

(c) Yaw

FIgure 11: Turntable test results.

To meet the requirement of rapid response, a fast compass alignment method based on data repeated calculation is designed. In this method, the same set of data is solved repeatedly to produce different DCM and different projected sensor data in mathematical platform from measured sensor data in body frame, thus producing different feedback signals. With the higher performance of navigation computer and less time for sensor data collecting, alignment time is significantly decreased.

Compared with the classical compass alignment, the results of turntable test can be summarized as follows: (1) the horizontal alignment accuracy is improved by 6 times and the azimuth alignment accuracy is improved by 8 times when the mean of the alignment is taken as the evaluation criteria and (2) the horizontal alignment accuracy is improved by 1 time and the azimuth alignment accuracy is improved by 4 times when the peak to peak value of the alignment curve is taken as the evaluation criteria. The alignment time measured by the computer with $333 \mathrm{MHz}$ frequency is $402 \mathrm{~s}$, which
TABLE 6: Statistics for turntable test results.

\begin{tabular}{|c|c|c|c|c|}
\hline & Scheme 1 & Scheme 2 & $\begin{array}{l}\text { Limit } \\
\text { value }\end{array}$ & $\begin{array}{c}\text { True } \\
\text { value }\end{array}$ \\
\hline \multicolumn{5}{|l|}{ Pitch $\left(\right.$ unit $\left.^{\circ}\right)$} \\
\hline Mean & 0.00855 & 0.00857 & 0.0585 & 0 \\
\hline Variance & 0.01558 & 0.01557 & & \\
\hline Peak-peak & $0.0291 /-0.0146$ & $0.0296 /-0.0142$ & & \\
\hline \multicolumn{5}{|c|}{ Roll (unit $\left.{ }^{\circ}\right)$} \\
\hline Mean & 0.00812 & 0.00814 & -0.0585 & 0 \\
\hline Variance & 0.01528 & 0.01531 & & \\
\hline Peak-peak & $0.0288 /-0.0144$ & $0.0290 /-0.0144$ & & \\
\hline \multicolumn{5}{|l|}{ Yaw (unit ${ }^{\circ}$ ) } \\
\hline Mean & 37.4951 & 37.4703 & 37.2135 & 37.5287 \\
\hline Variance & 0.00995 & 0.00996 & & \\
\hline Peak-peak & $37.5085 / 37.4673$ & $37.4818 / 37.4510$ & & \\
\hline
\end{tabular}


is slightly shortened in comparison with that in classical compass alignment.

\section{Acknowledgments}

This work was supported in part by the National Natural Science Foundation $(61004125,61273056)$ and Chinese university research and operation expenses (104.205.2.5).

\section{References}

[1] E. Levinson and C. S. Giovanni, "Laser gyro potential for long endurance marine navigation," in Proceedings of the IEEE Position Location and Navigation Symposium, pp. 115-129, Piscataway, NJ, USA, December 1980.

[2] E. Levinson, J. ter Horst, and M. Willcocks, "The next generation marine inertial navigator is here now," in Proceedings of the IEEE Position Location and Navigation Symposium, pp. 121-127, Las Vegas, Nev, USA, April 1994.

[3] J. I. Lahham and J. R. Brazell, "Acoustic noise reduction in the MK 49 ship's inertial navigation system (SINS)," in Proceedings of the IEEE Position Location and Navigation Symposium (PLANS '92), pp. 32-39, Charlottesville, Va, USA, March 1992.

[4] H. N. Weng, Q. C. Lu, K. Huang et al., "Rotation scheme design for rotary optical gyro SINS," Journal of Chinese Inertial Technology, vol. 17, no. 3, pp. 8-14, 2009 (Chinese).

[5] B. L. Yuan, Research on Rotating Inertial Navigation System with Four-Frequency Differential Laser Gyroscope, Graduate School of National University of Defense Technology, 2007.

[6] B. L. Yuan, D. Liao, and S. L. Han, "Error compensation of an optical gyro INS by multi-axis rotation," Measurement Science \& Technology, vol. 23, no. 2, pp. 1-9, 2012.

[7] Y. Yang and L. J. Miao, "Fiber-optic strapdown inertial system with sensing cluster continuous rotation," IEEE Transactions on Aerospace and Electronic Systems, vol. 40, no. 4, pp. 1173-1178, 2004.

[8] Y. N. Gao, J. B. Chen, and T. P. Yang, "Error analysis of strapdown optic fiber gyro compass," Transaction of Beijing Institute of Technology, vol. 25, no. 5, pp. 423-426, 2005.

[9] J. C. Yu, J. B. Chen, and J. H. Han, "Multiposition observability analysis of strapdown inertial navigation system," Transaction of Beijing Institute of Technology, vol. 24, no. 2, pp. 150-153, 2004 (Chinese).

[10] X. L. Wang and G. X. Sheng, "Fast and precision multiposition initial alignment method of inertial navigation system," Journal of Astronautics, vol. 23, no. 4, pp. 81-84, 2002 (Chinese).

[11] J. C. Fang and D. J. Wan, "A fast initial alignment method for strapdown inertial navigation system on stationary base," IEEE Transactions on Aerospace and Electronic Systems, vol. 32, no. 4, pp. 1501-1505, 1996.

[12] H. T. David and L. W. John, Strapdown Inertial Navigation Technology, Institute of Electrical Engineers, Hampshire, UK, 2nd edition, 2004.

[13] A. Jamshaid and J. C. Fang, "Alignment of strapdown inertial navigation system: a literature survey spanned over the last 14 years," ftp://labattmot.ele.ita.br/ele/alessandro/Leitura/ 04\%20-\%20PAPERS/ARTIGOS/KALMAN/kalmanlib/ StrapdownAlignment.pdf.

[14] Y. Li, X. X. Xu, and B. X. Wu, "Gyrocompass self-alignment of SINS," Journal of Chinese Inertial Technology, vol. 16, no. 4, pp. 386-389, 2008 (Chinese).
[15] X. Liu and X. Xu, "System calibration techniques for inertial measurement units," Journal of Chinese Inertial Technology, vol. 17, no. 5, pp. 568-571, 2009 (Chinese). 


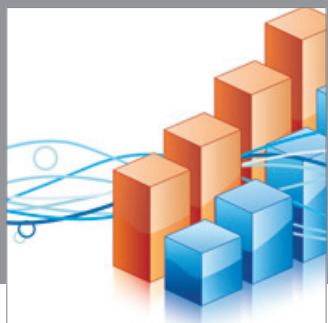

Advances in

Operations Research

mansans

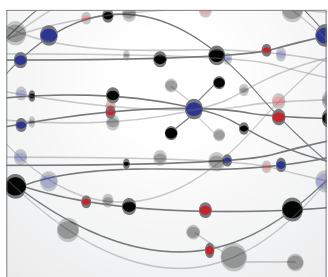

The Scientific World Journal
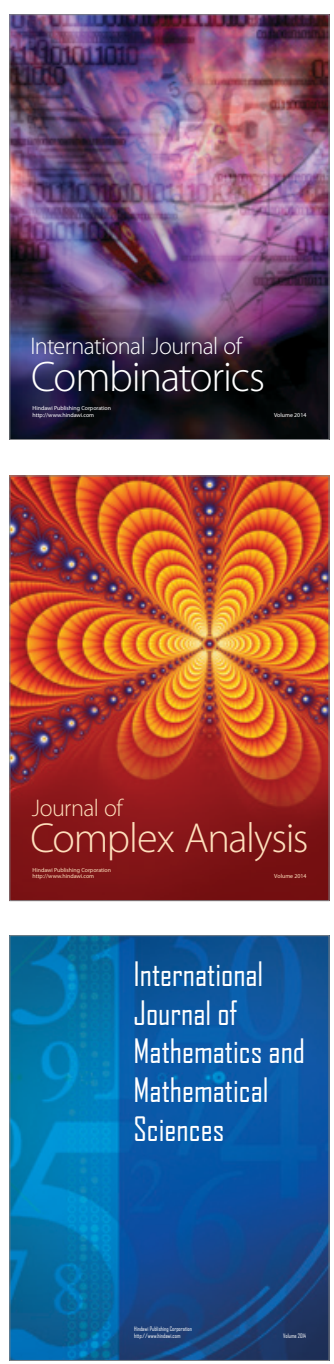
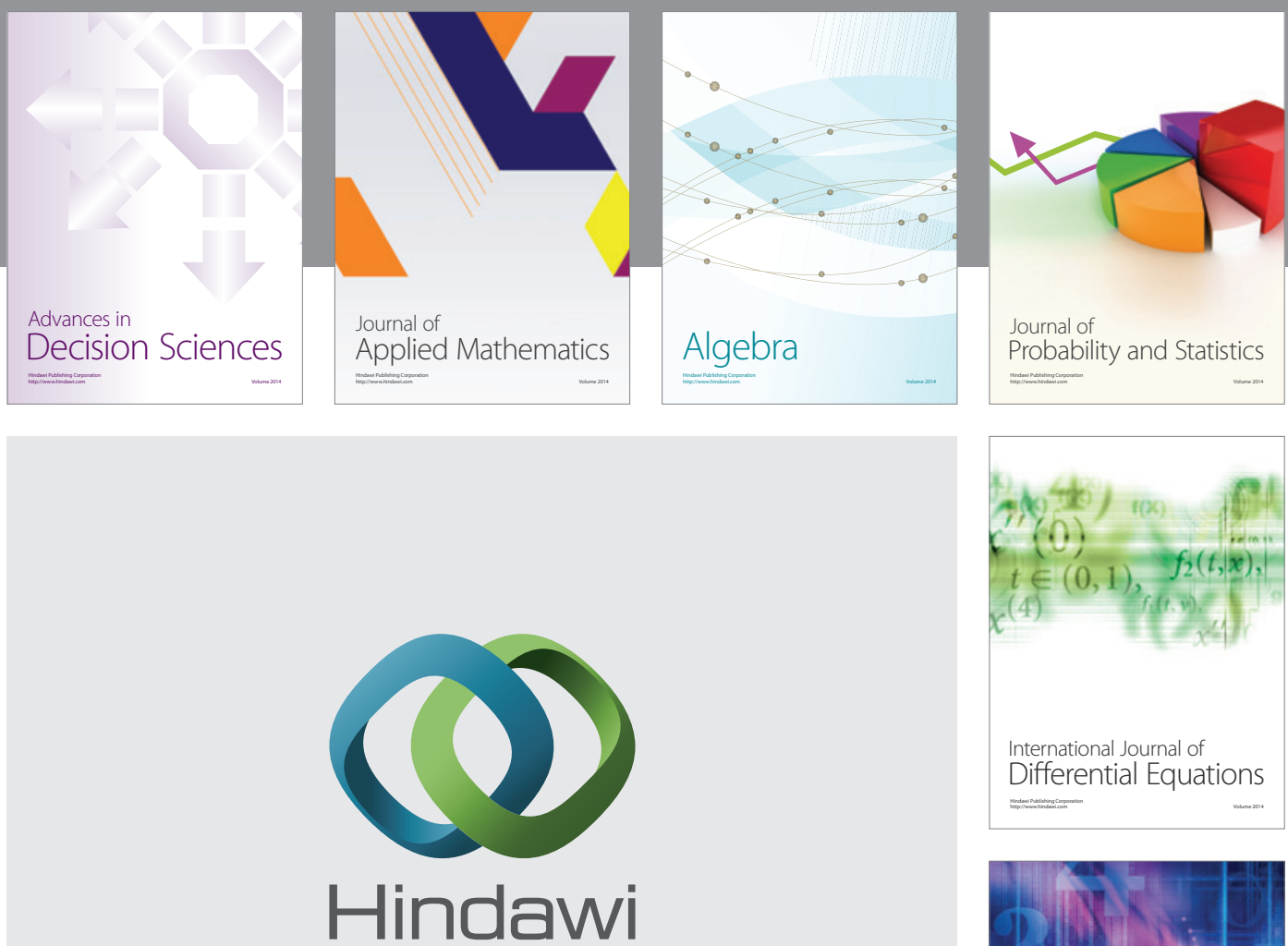

Submit your manuscripts at http://www.hindawi.com
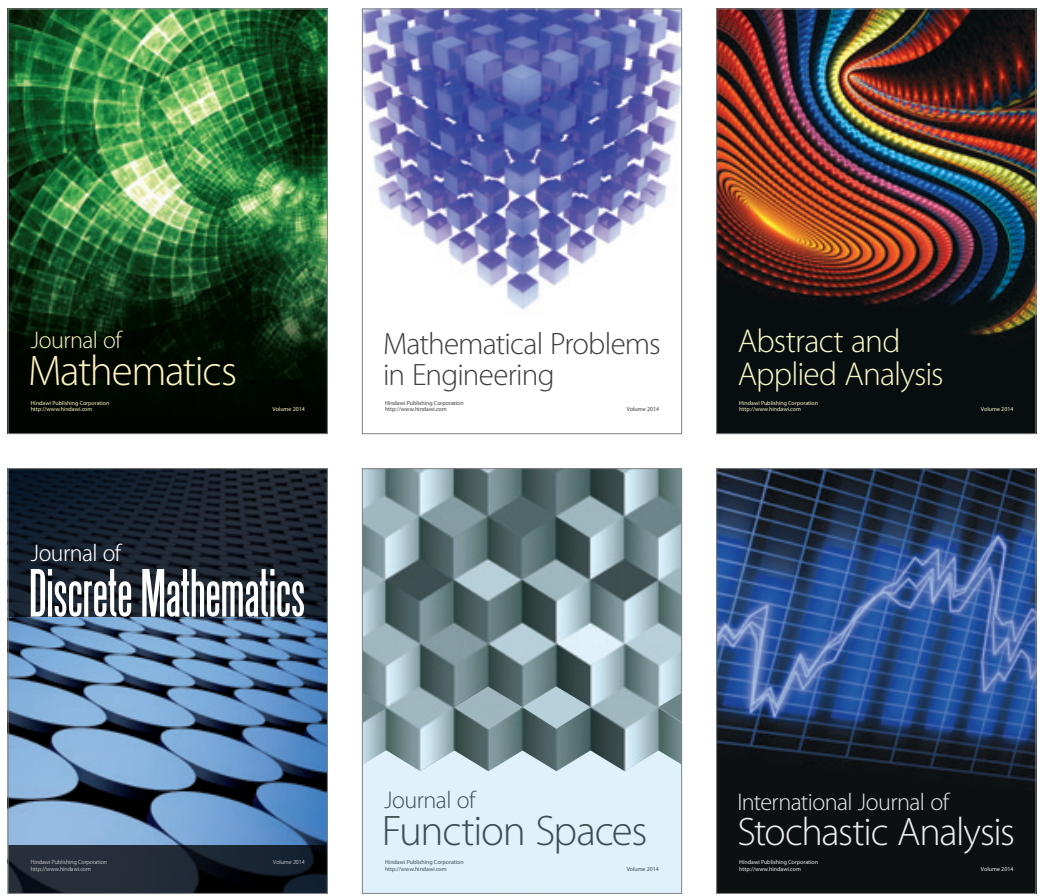

Journal of

Function Spaces

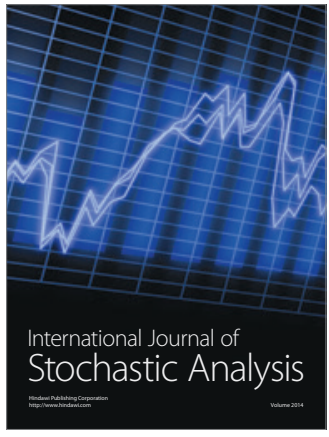

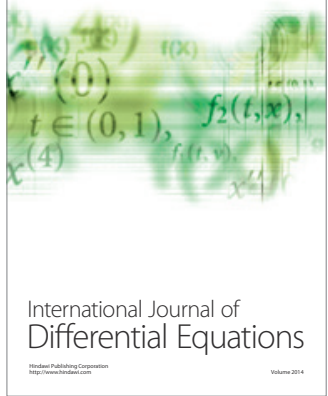
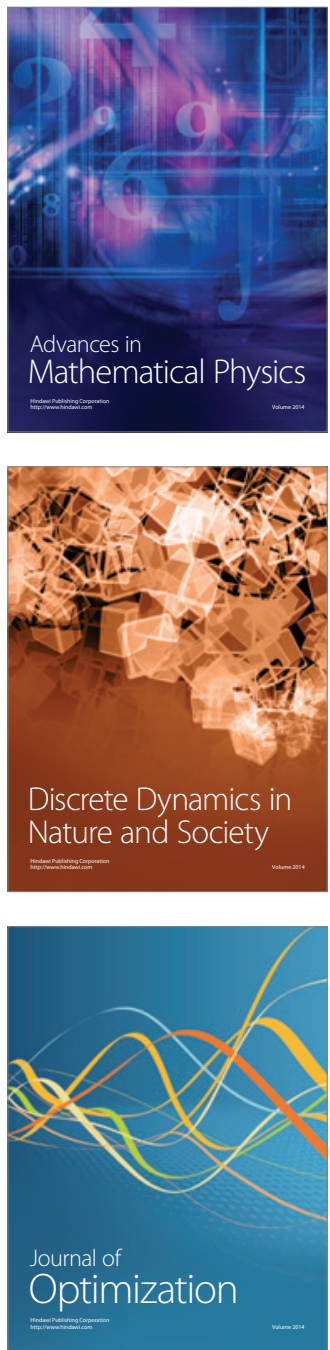